\title{
Effect of Molecular Weight on Viscoelastic Properties of Polymers as Predicted by a Molecular Theory
}

\author{
Hansjörg Oser and Robert S. Marvin
}

(February 27, 1963)

\begin{abstract}
Calculations have been made covering the predictions of a model representing the viscoelastic behavior of rubberlike polymers for molecular weights greater than $M_{c}, M_{c}$ being the lower limit of the range in which the viscosity is proportional to $M^{3.4}$. A pronounced difference in the character of $G^{\prime \prime}$ is predicted for polymers with molecular weights between five and ten times $M_{c}$ as compared with those whose molecular weights are outside this range.
\end{abstract}

In a recent publication [1] ${ }^{1}$ we presented the steady state and transient behavior predicted by a model designed to represent the mechanical behavior, in shear, of rubberlike polymers. This model is very similar to the molecular theory of Rouse [2], except that it includes directly the effect of entanglements found in long polymer chains.

Our model assumes an entropy-type elasticity as the origin of the relaxing force observed in a stressrelaxation experiment. It involves a resistance coefficient per unit segment of a polymer chain which is evaluated from the steady state viscous behavior, and the molecular weight of the polymer, expressed as the ratio of molecular weight to critical molecular weight, $M_{c}$, defined as the lower limit of the range in which the viscosity is proportional to $M^{3.4}$.

For the particular example we presented in reference 1 this ratio was 60 , and at the time we prepared this previous paper we did not have systematic calculations showing the predictions of the model for a range of molecular weights. We made an effort (sec. 3, p. 176 of reference (1)) to relate the extrema of $G^{\prime \prime}$ and $J^{\prime \prime}$ to the molecular weight through the use of various approximate relationships, since the full expressions can only be treated numerically. We have now carried out further numerical calculations covering a range of molecular weights, and find that our previous approximate relations were completely inadequate to represent the positions of these extrema as functions of molecular weight. In addition, we have found certain quite unexpected qualitative features of the predicted curves when the molecular weight is varied between one and ten times the critical molecular weight. Since these qualitative features may present the possibility of a rather critical test of the validity of this model, we present the results here in some detail.

The results of our previous paper may be represented in terms of normalized functions, the

1 Figures in brackets indicate the literature references at the end of this paper. normalized modulus,

$$
\left(5^{*}(\gamma)=c_{2} G^{*}=\left(S^{\prime}(\theta)+i\left(S^{\prime \prime \prime}(\theta)\right.\right.\right.
$$

and the normalized compliance,

$$
\Im^{*}(\gamma)=\frac{1}{c_{2}} J^{*}=\Im^{\prime}(\theta)-i \Im^{\prime \prime}(\theta)
$$

$c_{2}$ is a parameter equal to the kinetic theory equilibrium compliance of a vulcanized rubber with molecular weight $M_{c} / 2$ between cross links. Obviously, these are reduced functions since the ordinary temperature and density dependence are included in $c_{2} . \quad \gamma$ and $\theta$ are reduced frequencies, defined by

$$
\gamma=\theta(1+i)=\sqrt{i \omega c_{2} r}=\sqrt{\frac{\omega c_{2} r}{2}}(1+i)
$$

where $\omega$ is the actual radian frequency and $r$ the viscosity of a polymer of molecular weight $M_{c}$. In these terms,

$$
\mathfrak{F}^{*}(\gamma)=\gamma \frac{\tanh \gamma+L \tanh K \gamma}{1+L \tanh \gamma \tanh K \gamma}=\frac{1}{\mathcal{J}^{*}},
$$

where

$$
\begin{aligned}
L & =\left(\frac{l^{3 \cdot 4}-1}{l-1}\right)^{1 / 2}, \quad l=\frac{M}{M_{c}} \\
K & =L(l-1) .
\end{aligned}
$$

Equation (1) represents the predictions of our model up to frequencies where the modulus is within one or two decades of the limiting high frequency modulus. This range includes all the extrema which depend on molecular weight. The maximum in $G^{\prime \prime}$ occurring just before $G^{\prime}$ reaches its limiting or glassy value is independent of molecular weight. Its position and magnitude can be calculated easily as given in the previous paper [1].

The calculated values of the normalized modulus and compliance as a function of the normalized frequency, $\theta$, are shown for several values of $l$, used here as a measure of molecular weight, in figures 


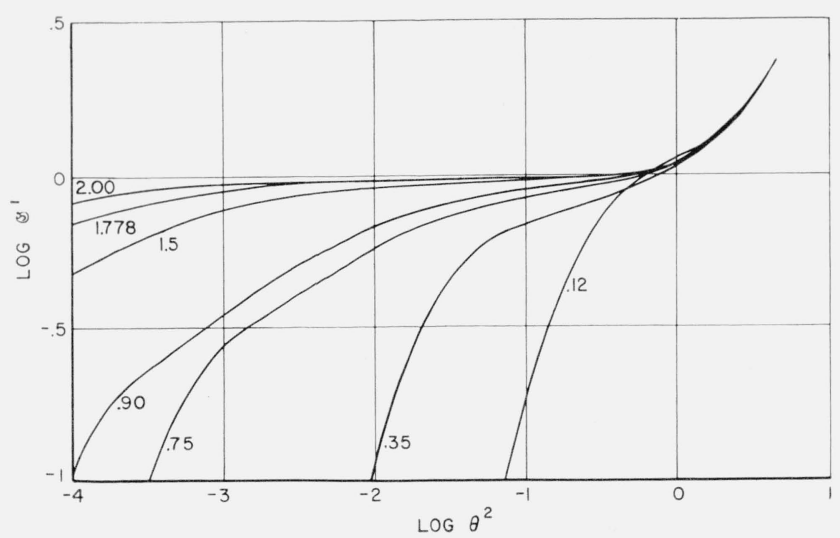

FIGURE 1. Real part of dynamic modulus for various molecular weights.

Parameters are values of $\log l=\log \left(M / M_{c}\right)$.

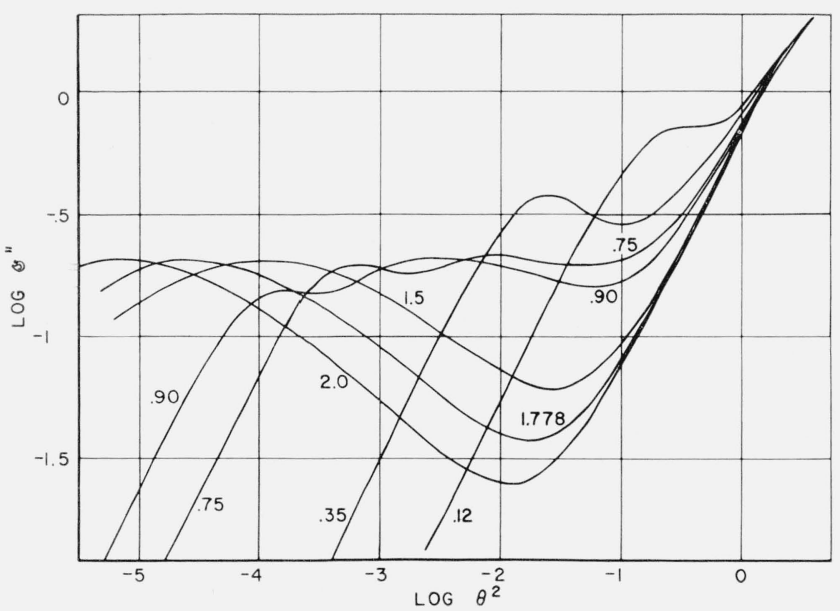

Figure 2. Imaginary part of dynamic modulus for various molecular weights.

Parameters are values of $\log l=\log \left(M / M_{c}\right)$

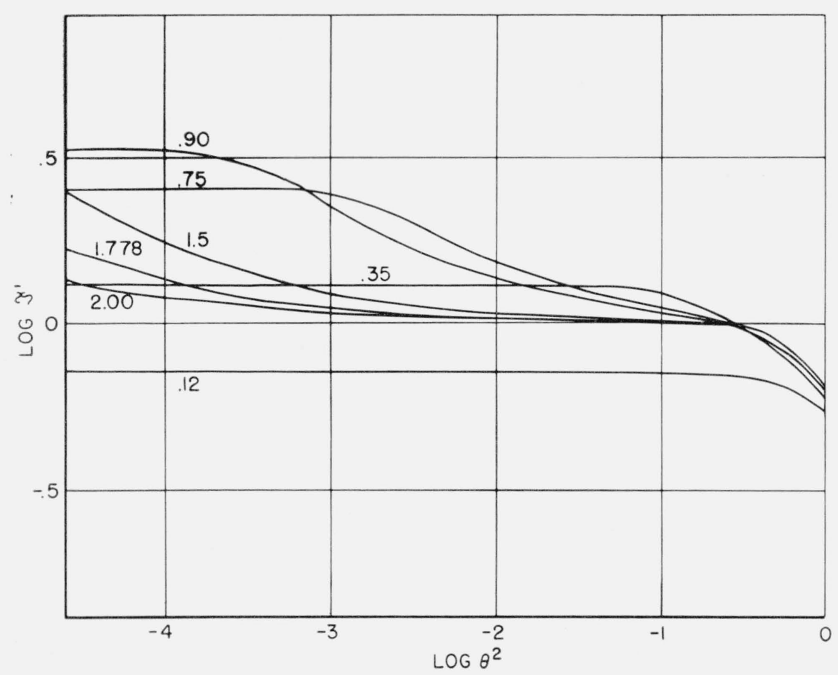

Figure 3. Real part of dynamic compliance for various molecular weights.

Parameters are values of $\log l=\log \left(M / M_{c}\right)$.

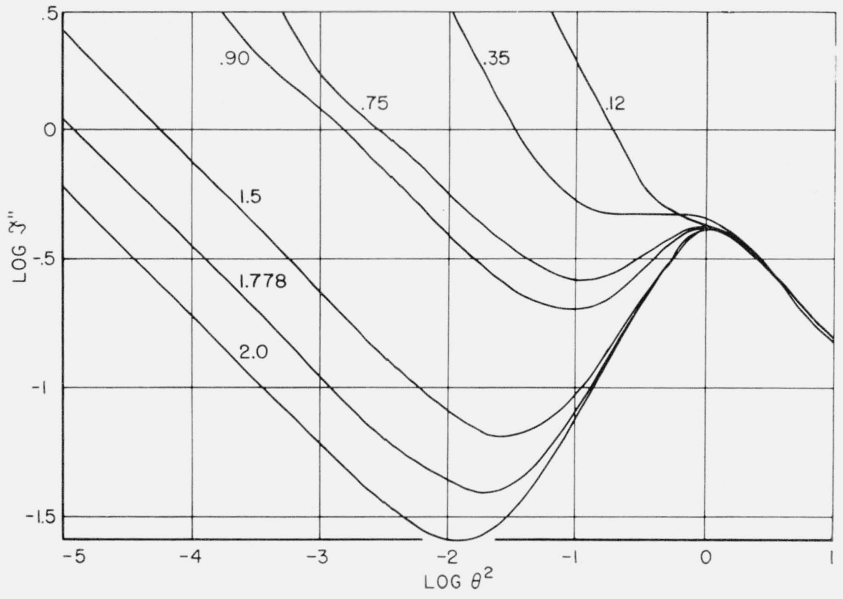

FIgure 4. Imaginary part of dynamic compliance for various molecular weights.

Parameters are values of $\log l=\log \left(M / M_{c}\right)$.

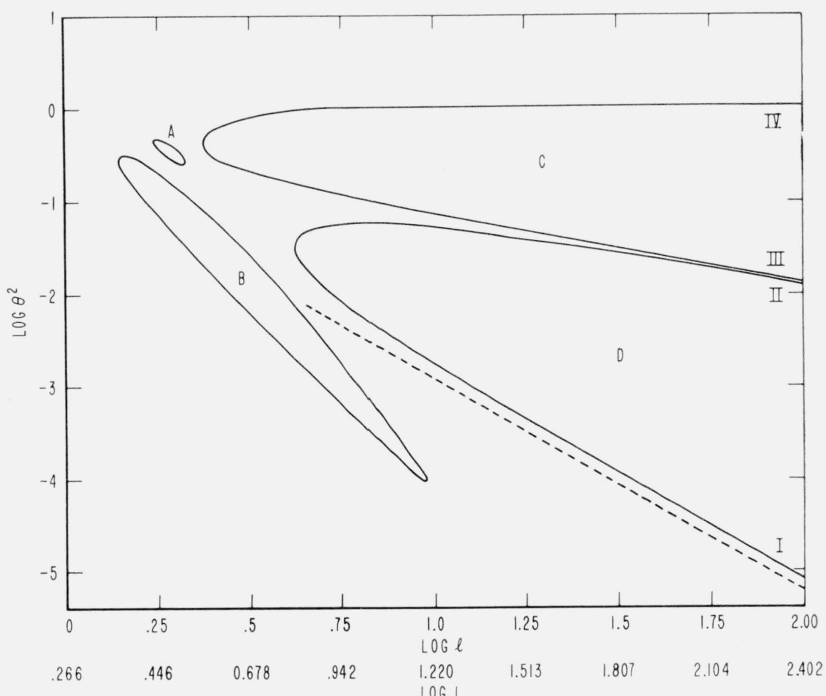

Figure 5. Reduced frequencies of the extrema as a function of

$$
l=\frac{\mathrm{M}}{\mathrm{M}_{\mathrm{c}}} .
$$

1 to 4 . We see that $\left(\mathfrak{S}^{\prime \prime}\right.$ and $\mathcal{S}^{\prime \prime}$ do not show any extrema for certain values of $l$, and that for other values of $l$ there are two minima and two maxima in $(5)$ below the glass y range.

This occurrence of two sets of extrema was certainly unexpected. To illustrate this behavior more clearly, we show in figure 5 a plot of $\theta_{\text {ext }}$, the frequencies at which the extrema occur, as a function of $l$ and $L$. We distinguish four separate curves $A$ and $C$ belonging to $\mathcal{F}^{\prime \prime}(\theta), B$ and $D$ belonging to $\left(5^{\prime \prime}(\theta)\right.$. The lower branches of $A$ and $C$ represent at the minimum of $\Im^{\prime \prime}$, the upper branches $\theta$ at the maximum of that function. The lower branches of the curves $B$ and $D$ represent $\theta$ at the maximum of (5)" and the upper branches give $\theta$ at the minimum of that function. 
The points with vertical tangents (fig. 5) correspond to inflection points of $\left(\xi^{\prime \prime}\right.$ or $\mathcal{S}^{\prime \prime}$ with a horizontal tangent. This follows immediately, once we realize that they represent points where a maximum and minimum have coalesced.

It is interesting to examine the asymptotic behavior of the branches of $C$ and $D$, for $l \rightarrow \infty$, since here these curves apparently become straight lines and we expect simple power laws for the functional relation between $\theta$ and $l$ (or $L$ ). We observe from figure 5 that a common lower bound for all four branches of the curves $C$ and $D$ is given by

$$
\theta=\frac{1}{2 l^{1.2}} \sim \frac{1}{2 L}
$$

as indicated by the dotted line in figure 5. Hence

$$
\operatorname{Re}(K \gamma)=K \theta>\frac{K}{2 L}=\frac{l-1}{2}>4.5
$$

in the region $l \geq 10, L \geq 16.5$.

This inequality shows that tanh $(K \gamma)$ in the expressions for $(\$)^{\prime \prime}$ and $\mathcal{S}^{\prime \prime}$ can be put equal to unity and therefore does not influence the location of any of the extrema for large $l$. Our functions simplify then as

follows, if we introduce $\chi=\tanh ^{-1}\left(\frac{1}{L}\right)$

$$
(5)^{\prime \prime}(\theta)=\operatorname{Im}\{\gamma \operatorname{coth}(\gamma+\chi)\}=\theta \frac{\sinh 2(\theta+\chi)-\sin 2 \theta}{\cosh 2(\theta+\chi)-\cos 2 \theta}
$$

$\Im^{\prime \prime}(\theta)=-\operatorname{Im}\left\{\frac{1}{\gamma} \tanh (\gamma+\chi)\right\}$

$$
=\frac{1}{2 \theta} \frac{\sinh 2(\theta+\chi)-\sin 2 \theta}{\cosh 2(\theta+\chi)+\cos 2 \theta}
$$

We turn now to the determination of the asymptotic behavior of the branches I, II, III, IV. On both branches I and II of curve $D, \theta$ and $\chi$ approach zero as $L$ increases. We can thus use Taylor's expansion to obtain:

$$
\left(\mathfrak{S}^{\prime \prime}=\frac{\theta \chi}{2 \theta^{2}+2 \chi \theta+\chi^{2}}+\frac{1}{3} \theta(2 \theta+\chi)+O\left(\theta^{3}\right) .\right.
$$

The derivative of the first term (with respect to $\theta$ ) predicts a maximum at $\theta_{\max }=\frac{\chi}{\sqrt{2}}=\frac{1}{2^{1 / 2} L}\left(\chi \approx \frac{1}{L}\right.$ for large $\left.L\right)$ with $\mathfrak{S}_{\max }^{\prime \prime}=0.207$. This is the only extremum predicted by the first term alone. To find a condition for the minimum we re-write the above as:

$$
\left(5^{\prime \prime}\right)=\frac{\chi}{2 \theta}\left(1-\frac{\chi}{\theta}+\frac{\chi^{2}}{2 \theta^{2}}+\ldots\right)+\frac{2}{3} \theta^{2}\left(1+\frac{\chi}{2 \theta}\right)+O\left(\theta^{3}\right),
$$

this expression being permitted because on branch II $\chi \ll \theta^{2}$. This latter relation justifies our taking, for large $L$,

$$
\text { (3)' } \approx \frac{\chi}{2 \theta}+\frac{2}{3} \theta^{2} \text {. }
$$

From which we predict a minimum at

$$
\theta_{\min }=\left(\frac{3}{8 L}\right)^{1 / 3}
$$

with a magnitude

$$
\mathfrak{S S}_{\min }^{\prime \prime}=1.04 L^{-2 / 3} .
$$

An expression for the asymptote of the minimum in $\mathfrak{J}^{\prime \prime}$ represented by branch III (curve $C$ ) may be obtained from a similar expansion of tanh $(\gamma+\chi)$, again retaining two terms and using the fact that $\chi<\theta^{2}$. It yields, as is apparent from figure 5 , the same value as the asymptote for branch II:

$$
\Im^{\prime \prime} \approx \frac{\chi}{2 \theta}+\frac{2}{3} \cdot \theta^{2},
$$

from which

$$
\theta_{\min }=\left(\frac{3}{8 L}\right)^{1 / 3}
$$

and

$$
\Im_{\min }^{\prime \prime}=1.04 L^{-2 / 3}
$$

The maximum in $\mathcal{I}^{\prime \prime}$, branch IV of curve $C$, appears to be nearly independent of $l$. If it were strictly independent, it would be the maximum of:

$$
\frac{1}{2 \theta} \cdot \frac{\sinh 2 \theta-\sin 2 \theta}{\cosh 2 \theta+\cos 2 \theta}
$$

the asymptotic expression corresponding to eq (3) for large $l$. This function has a maximum value of

at

$$
\Im_{\max }^{\prime \prime}=0.4172
$$

$$
\theta_{\max }=1.127 \text {. }
$$

The following tabulation shows that these maxima of our calculated $\Im^{\prime \prime}$ functions depend only very slightly on $l$ :

$$
\begin{aligned}
& \text { for } l=5.012: \Im_{\max }^{\prime \prime}=0.4325, \theta_{\max }=1.019 \\
& \text { for } l=100.00: \Im_{\max }^{\prime \prime}=0.4178, \theta_{\max }=1.122 .
\end{aligned}
$$

It is difficult to do more than point out the existence of curves $A$ and $B$ (fig. 5), which was not even suspected prior to these detailed numerical calculations. Since in this region tanh $K \gamma$ cannot be taken as unity, the analytic expressions for $\left(\mathfrak{b}^{\prime \prime}\right.$ and $\mathcal{G}^{\prime \prime}$ are very complicated. There is no reason to expect that expressions for curves $A$ and $B$ would be simple enough to be useful. Therefore we leave them with the presentation of the numerical results. These computations have now been carried out in sufficient detail that we are essentially certain we have found all the extrema predicted by the model for both $\left(5^{\prime \prime}\right.$ and $\Im^{\prime \prime}$. 
The behavior of $\left(\mathrm{H}^{\prime}\right)$ and $\left(\mathrm{S}^{\prime \prime \prime}\right.$ calculated here as a function of molecular weight should provide a critical test of our model if appropriate experimental data could be found for comparison. In the molecular weight range of about 5 to 10 times $M_{c}$ this model predicts an essentially constant value of $G^{\prime \prime}$ over two to three decades of frequency, with $G^{\prime}$ increasing fairly steadily over the same range. For higher molecular weights the $G^{\prime \prime}$ curve should show a pronounced maximum and minimum, their separation becoming greater on both the frequency and modulus scales with increasing molecular weight. In the vicinity of the minimum of $G^{\prime \prime}, G^{\prime}$ should remain fairly constant. Since we do not know how a distribution of molecular weights should be introduced into our model, it would be preferable to make the comparison with experimental measurements on fractionated materials, but lacking such data it seems most reasonable to use a weight average molecular weight.

While we do not know of any dynamic measurements which can be used to check these predictions directly, the stress relaxation measurements and calculated relaxation spectra reported by Tobolsky and Murakami [3] for a series of polystyrene samples with weight average molecular weights ranging from $4.25 \times 10^{4}\left(=1.1 M_{c}\right)$ to $3.15 \times 10^{5}\left(=8.3 M_{c}\right)$ (their sample numbers 6 to 15 ) and $\bar{M}_{v} / \bar{M}_{n}=1.5$ represent a reasonable check on the predictions summarized above. The stress relaxation modulus is approximately the mirror image of $G^{\prime}$, and the spectrum $\bar{H}(\tau)$ is, to a zero order approximation, equal to $(2 / \pi) G^{\prime \prime}(1 / \tau)$.

The graphs of $\bar{H}$ shown in figure 4 of reference 3 show no plateau region for molecular weights between $M_{c}$ and $2.4 M_{c}$ (samples 6 to 11 ). Distinct plateau regions are seen for samples with molecular weights between $3.2 M_{c}$ and $8.3 M_{c}$ (samples 12 to 15 ). For all these samples the $G(t)$ curves are monotonically decreasing, with no hint of a plateau region. For higher molecular weights, of course, we normally find a region where $G(t)$ or $G^{\prime}(\omega)$ are changing so slowly as to suggest strongly a plateau. This type of behavior is observed in our calculated curves for $l>15$.

\section{References}

[1] R. S. Marvin and H. Oser, J. Res. NBS, 66B (Math. and Math. Phys.) No. 4, 171 (1962).

[2] P. E. Rouse, Jr., J. Chem. Phys. 21, 1272 (1953).

[3] A. V. Tobolsky and K. Murakami, J. Polymer Sci. 47, $55(1960)$.

(Paper 67B2-96) 